\title{
The role of qSOFA score and biomarkers in assessing severity of community-acquired pneumonia in adults
}

\author{
Raluca-Elena Tripon ${ }^{1 *}$, Victor Cristea ${ }^{2}$, Mihaela-Sorina Lupse ${ }^{3}$ \\ 1. Department of Infectious Diseases, "Iuliu Hatieganu” University of Medicine and Pharmacy; \\ Teaching Hospital of Infectious Diseases, Romania \\ 2. Department of Immunology, "Iuliu Hatieganu" University of Medicine and Pharmacy, Romania \\ 3. Department of Infectious Diseases, "Iuliu Hatieganu” University of Medicine and \\ Pharmacy; Teaching Hospital of Infectious Diseases, Romania
}

\begin{abstract}
Introduction: Community-acquired pneumonia (CAP) is the primary cause of severe sepsis. Severity assessment scores have been created, in order to help physicians decide the proper management of CAP. The purpose of this study was to examine the correlations between different CAP severity scores, including qSOFA, several biomarkers and their predictive value in the 30 day follow-up period, regarding adverse outcome. Materials and methods: One hundred and thirty nine adult patients with CAP, admitted in the Teaching Hospital of Infectious Diseases, Cluj-Napoca, Romania from December 2015 to February 2017, were enrolled in this study. Pneumonia Severity Index (PSI), CURB-65, SMART-COP and the qSOFA scores were calculated at admittance. Also, C-reactive protein (CRP), procalcitonin (PCT) and albumin levels were used to determine severity. Results: The mean PSI of all patients was $93.30 \pm 41.135$ points, for CURB-65 it was $1.91 \pm 0.928$ points, for SMART-COP it was $1.69 \pm 1.937$ points. The mean qSOFA was $1.06 \pm 0.522$ points, 21 (14.9\%) were at high risk of in-hospital mortality. In the group of patients with qSOFA of $\geq 2$, all pneumonia severity scores and all biomarkers tested were higher than those with scores $<2$. We found significant correlations between biomarkers and severity scores, but none regarding adverse outcome. Conclusion: The qSOFA score is easier to use and it is able to accurately evaluate the severity of CAP, similar to other scores. Biomarkers are useful in determining the severity of the CAP. Several studies are needed to assess the prediction of these biomarkers and severity scores in pneumonia regarding adverse outcome.
\end{abstract}

Keywords: biomarkers, albumin, community-acquired pneumonia, qSOFA, pneumonia severity scores Received: $3^{\text {rd }}$ July 2020; Accepted: $1^{\text {st }}$ November 2020; Published: $2^{\text {nd }}$ January 2021

\footnotetext{
* Corresponding authors: Tripon Raluca-Elena, Department of Infectious Diseases, "Iuliu Hatieganu” University of Medicine and Pharmacy; Teaching Hospital of Infectious Diseases, Cluj-Napoca, Romania. E-mail: rhorge@yahoo.com
} 


\section{Introduction}

Despite the advances in diagnostic methods and treatment, community-acquired pneumonia (CAP) remains one of the world's major causes of morbidity and mortality (1). Also, it is the primary cause of severe sepsis $(1,2)$.

It is very important for physicians to decide the proper management of CAP, so several severity assessment scores have been created in the last decades, in order to help them. Pneumonia severity index (PSI) is the most favoured and established severity scoring method measuring mortality risk in patients with pneumonia, but it is also very complex and hard to use in daily practice (3). Therefore, the CURB-65 score was proposed as a simplified alternative (4). Several other prognostic tools have been developed, such as SMART-COP for assessing the need of intensive respiratory and vasopressor support (IRVS) (5). Also, biomarkers have been proposed to predict severity in CAP. The level of serum $\mathrm{C}$ - reactive protein (CRP) is considered to be a useful marker for establishing the diagnosis and severity of CAP in adults (6). Procalcitonin (PCT) is also a well-studied biomarker for predicting severity in CAP, and studies have found it can be used accurately in clinical practice, to guide antibiotic therapy (7).Serum albumin was examined in critically ill patients showing that it is an indicator of inflammation rather than a nutritional status indicator (8). Lower plasma albumin concentrations in CAP seemed to be a strong predictor of mortality (8).

Using the quick Sequential Organ Failure Assessment (qSOFA), the sepsis concept was revised for a better classification of patients at high risk of death (9). A qSOFA score of $\geq 2$ plus a suspected infection was recommended to help classify patients with possible sepsis outside of the intensive care unit (ICU) (10). Pneumonia is a major cause of sepsis, therefore, it is important to determine the outcome prediction of the
qSOFA score in these patients. A recent Japanese research used SOFA and qSOFA scores in combination, in order to evaluate the severity of Healthcare associated pneumonia (HCAP) and CAP (11).

In this study, we investigated the correlations between different severity scores in CAP, including qSOFA and their predictive value regarding adverse outcome, consisting in relapses and death. We also investigated the relationship between severity scores and several markers like C-reactive protein (CRP), procalcitonin (PCT) and albumin levels and their predictive value regarding adverse outcome. The association between adverse outcome and several interleukin levels in CAP has been studied elsewhere (12).

\section{Method}

This observational study was conducted in the Teaching Hospital of Infectious Diseases Cluj-Napoca, Romania, between December 2015 and February 2017 and included adult patients with CAP defined according to the IDSA (13). This work was approved in compliance with the ethical standards of the Helsinki Declaration, by the medical ethics committee of both the Hospital and "Iuliu Hatieganu" University of Medicine and Pharmacy Cluj-Napoca, Romania. A written informed consent was obtained from patients or their closest relatives in each case. Exclusion criteria were represented by: age under 18 years old, pregnancy, HIV infection, active tuberculosis and autoimmune diseases requiring immunosuppressive drugs. Patients were enrolled in the study group within 24 hours of hospital admission. We recorded: demographical data, clinical data, length of stay (LOS) in hospital, presence of complications such as: sepsis, according to the ACCP/SCCM and SEPSIS 3 criteria $(10,14)$, acute respiratory failure and pleurisy. 
The follow-up period was 30 days after discharge. We recorded adverse outcomes during this period, meaning relapses and death.

At admission, we evaluated microorganisms isolated from sputum and/or blood cultures. Sputum processing involved performing a Gram stain and culturing on culture media such as blood agar, chocolate agar and MacConkey agar. Subsequently, the identification method used was MALDI TOF mass spectrometry using MALDI Biotyper (Brucker). We also performed a serological test to detect antibodies against Mycoplasma pneumoniae and Chlamydia pneumoniae. Also, the rapid influenza diagnostic test was used when necessary, during the winter outbreaks. Blood samples were taken after admission to determine standard analytical parameters. CRP and serum albumin concentrations were analysed using the CRPL3 package in the COBAS C311 module analyser (Roche/Hitachi) from serum turbidimetric immunoassay with latex particles coated with anti-CRP, monoclonal antibodies. Normal values for CRP were $0-1 \mathrm{mg} /$ $\mathrm{dl}$, and for albumin 3.2-5.2 g/dl.

PCT levels were analysed from serum using the one-step immunoassay sandwich method with a final fluorescent detection (ELFA), by VIDAS BRAHMS (bio Mérieux). Low risk for sepsis was considered $<0.5 \mathrm{ng} / \mathrm{dl}$.

The Pneumonia Severity Index (PSI) (3), CURB-65 (4), SMART-COP (5) as scores for pneumonia severity, and the qSOFA score, were determined upon admission (9).

\section{Statistical analysis}

Discrete variables were expressed in parentheses as counts (percentage) and continuous variables as means, \pm standard deviation (SD) or median and interquartile range (IQ), unless otherwise specified. The Chi-square test was used to compare frequency. The Student $t$ test was necessary in order to reveal a two-group comparison of the normally distributed results. The Mann-Whitney U test was used for data not normally distributed, where only two groups were compared and the Kruskal-Wallis one-way analysis of variance was used when more than two groups were compared. Correlation analyses were conducted using the Spearman rank correlation. All tests were calculated using IBM SPSS version 20.0 (IBM Corp, NY, USA). The receiver - operator curves (ROC) with the value level set at $p<0.05$, were used to evaluate prediction accuracy by estimating the area under the curve. The ROC curves were generated by MedCalc Statistical Software version 19.1.3 (Ostend, Belgium).

\section{Results}

A total of 139 adult patients needing hospitalization due to CAP were included. Baseline features of the study population are summarized in Table I.

The average LOS in hospital was $7.35 \pm 3.41$ days with no difference between gender $(p=$ $0.458)$ or age $(p=0.892)$.

Sputum cultures were performed in 74/139 patients $(53.23 \%)$. Blood cultures were performed in $58 / 139$ patients $(41.72 \%)$, but they were all negative. The etiology was established in 33 (24\%) cases: 2- Streptococcus pneumonia, 4Haemophilus influenzae, 1- Haemophilus parainfluenzae, 6- Chlamydia pneumoniae, 6- Mycoplasma pneumoniae, 12- Influenza virus (type A and B) during winter outbreaks, 1-Serratia marcescens (from community) and 1-E-coli (the patient died).

Regarding complications, 24 (17.3\%) patients had respiratory sepsis, 33 (23.7\%) had pleurisy, $1(0.7 \%)$ had empyema and $119(85.6 \%)$ had different degrees of respiratory failure.

Out of the 139 cases, 7 (5\%) required admission in the ICU, 2 requiring mechanical ventilation and dying in hospital. 
Table I. Baseline characteristics of the 139 patients

\begin{tabular}{|c|c|}
\hline \multicolumn{2}{|l|}{ Characteristics } \\
\hline Age, years & $58.73 \pm 18.84^{\mathrm{a}}$ \\
\hline Male gender, no. (\%) & $79(56.8)$ \\
\hline Urban areas, no. $(\%)$ & $80(57.6)$ \\
\hline \multicolumn{2}{|l|}{ Smoking status } \\
\hline Current smoker, no. (\%) & $33(23.7)$ \\
\hline Past smokers, no. (\%) & $35(25.2)$ \\
\hline No smokers, no. $(\%)$ & $71(51.0)$ \\
\hline Toxic environment, no (\%) & $3(2.2)$ \\
\hline Antibiotic pretreatment & $33(23.7)$ \\
\hline \multicolumn{2}{|l|}{ Comorbidies, no. $(\%)$} \\
\hline Hypertensive heart disease & $74(53.2)$ \\
\hline Coronary artery disease & $53(38.1)$ \\
\hline Congestive heart failure & $30(21.6)$ \\
\hline Cerebrovascular disease & $20(14.4)$ \\
\hline Renal dysfunction & $11(7.9)$ \\
\hline Liver disease & $11(7.9)$ \\
\hline Diabetes mellitus & $17(12.2)$ \\
\hline Chronic obstructive pulmonary & $15(10.6)$ \\
\hline \multicolumn{2}{|l|}{ disease } \\
\hline \multirow{2}{*}{\multicolumn{2}{|c|}{ History, no. (\%) }} \\
\hline & \\
\hline Fever & $119(85.0)$ \\
\hline Dry cough & $53(37.9)$ \\
\hline Productive cough & $68(48.6)$ \\
\hline Dyspnea & $74(52.9)$ \\
\hline \multicolumn{2}{|l|}{ Laboratory findings } \\
\hline $\mathrm{CRP}(\mathrm{mg} / \mathrm{dl})$, median (IQ range) & $11.15[17.45]$ \\
\hline Procalcitonin $(\mu \mathrm{g} / \mathrm{ml})$, median (IQ range) & $0.08[1.26]$ \\
\hline Albumin (g/dl) median (IQ range) & $3.75[0.68]$ \\
\hline \multicolumn{2}{|l|}{ Radiographic findings, no. (\%) } \\
\hline Pleural effusion & $34(24.5)$ \\
\hline Opacities & $83(59.7)$ \\
\hline Interstitial infiltrate & $20(14.4)$ \\
\hline Multilobar opacities & $23(16.4)$ \\
\hline \multicolumn{2}{|l|}{ PSI class, no. $(\%)$} \\
\hline I & $21(15.1)$ \\
\hline II & $16(11.5)$ \\
\hline III & $32(23.0)$ \\
\hline IV & $47(33.8)$ \\
\hline $\mathrm{V}$ & $23(16.5)$ \\
\hline \multicolumn{2}{|l|}{ CURB-65 groups, no. (\%) } \\
\hline 1 & $45(32.4)$ \\
\hline 2 & $62(44.6)$ \\
\hline 3 & $32(23.0)$ \\
\hline \multicolumn{2}{|l|}{ SMART-COP class, no. $(\%)$} \\
\hline 1 & $103(74.1)$ \\
\hline 2 & $19(13.7)$ \\
\hline 3 & $11(7.9)$ \\
\hline 4 & $6(4.3)$ \\
\hline \multicolumn{2}{|l|}{ qSOFA score } \\
\hline$<2$ & $118(83.7)$ \\
\hline$\geq 2$ & $21(14.9)$ \\
\hline
\end{tabular}


During the 30-day follow-up period, 23 patients $(16.5 \%)$ had adverse outcome, consisting in relapses ( 21 cases) and/or death ( 2 cases).

We found that the qSOFA score corresponds with certain severity scores for pneumonia, meaning CURB-65 and SMART-COP. We ob- served substantial associations between CRP rates and CURB-65, SMART-COP and qSOFA, but not PSI, while PCT was associated with all the severity scores. We found that lower albumin levels correlate with all severity scores. Also, biomarkers correlate with each other (Table II)

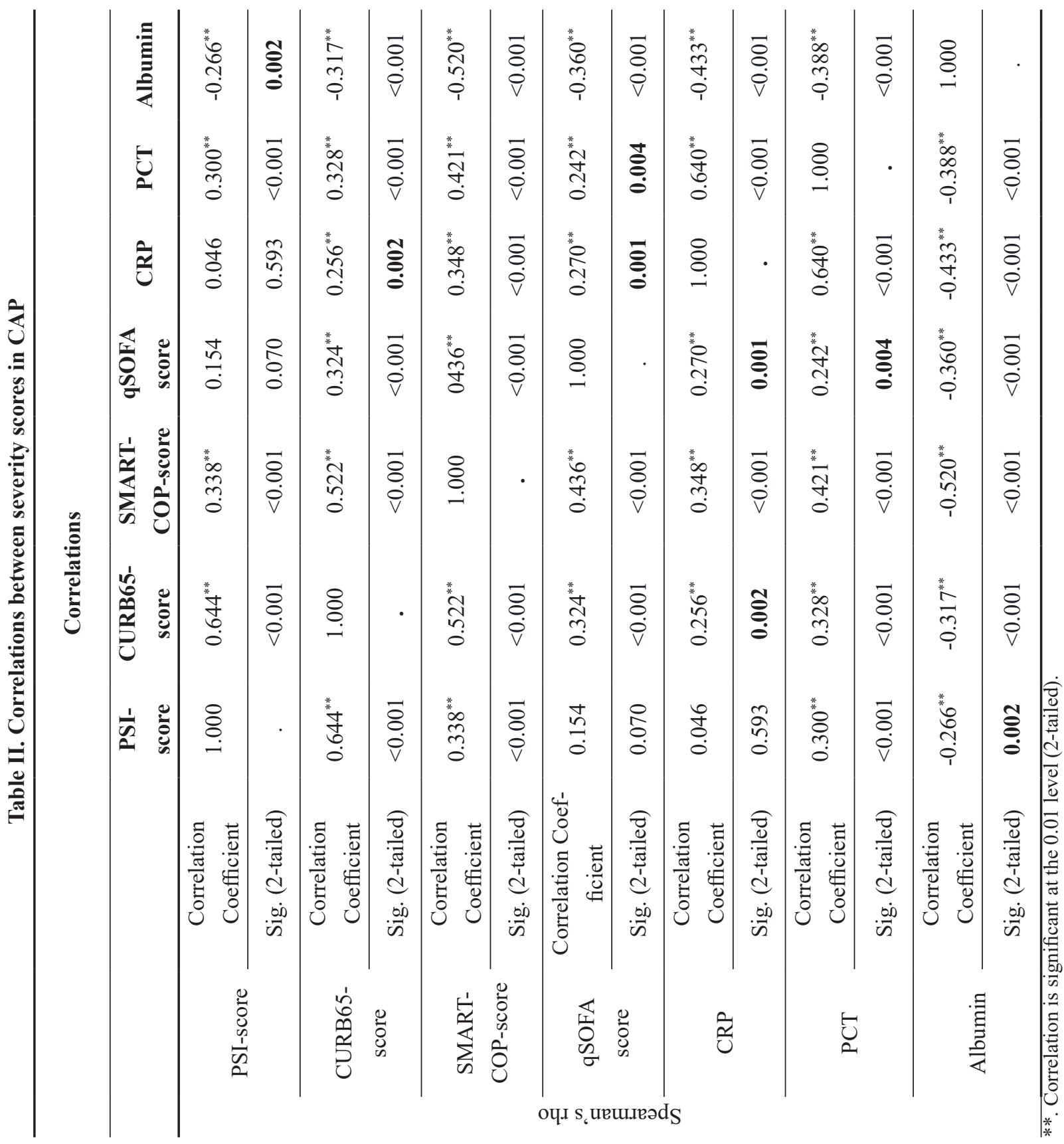


We divided the patients according to the qSOFA score into 2 groups: $<2$ and $\geq 2$ and we looked for correlations with other severity scores and with the studied biomarkers. The results were that the pneumonia severity scores, CRP and PCT were higher in the group of patients with a qSOFA of $\geq 2$ compared to those with scores of $<2$, while lower albumin levels were found in the $\mathrm{qSOFA} \geq 2$ group (Table III).
We investigated the capacity of the studied CAP prognostic scores and biomarkers included in this study to predict adverse outcome. For our patients, the area under the receiver operating characteristic curve (AUC) for PSI, CURB-65, SMART-COP and qSOFA scores were 0.681 $(\mathrm{p}=0.006), 0.586(\mathrm{p}=0.191), 0.645(\mathrm{p}=0.028)$ and $0.542(\mathrm{p}=0.527)$, respectively (Figure 1$)$.

Table III. Correlations between qSOFA and other pneumonia severity scores and biomarkers among CAP patients

\begin{tabular}{lccc}
\hline & qSOFA $<\mathbf{2}(\mathbf{n}=\mathbf{1 1 8})$ & $\mathbf{q S O F A} \geq \mathbf{2}(\mathbf{n}=\mathbf{2 1})$ & $\mathbf{p}$-value \\
\hline PSI $($ mean \pm SD) & $91.16 \pm 38.19$ & $105.33 \pm 54.40$ & 0.022 \\
\hline CURB-65 $($ mean \pm SD) & $1.83 \pm 0.86$ & $2.38 \pm 1.161$ & 0.003 \\
\hline SMART-COP $($ mean \pm SD) & $1.42 \pm 1.73$ & $3.24 \pm 2.30$ & 0.000 \\
\hline PCT $($ mean \pm SD) & $1.61 \pm 4.76$ & $4.12 \pm 9.81$ & 0.000 \\
\hline CRP $($ mean \pm SD) & $11.86 \pm 10.11$ & $15.91 \pm 11.26$ & 0.003 \\
\hline Albumin $($ mean \pm SD) & $3.70 \pm 0.56$ & $3.12 \pm 0.76$ & 0.000 \\
\hline
\end{tabular}

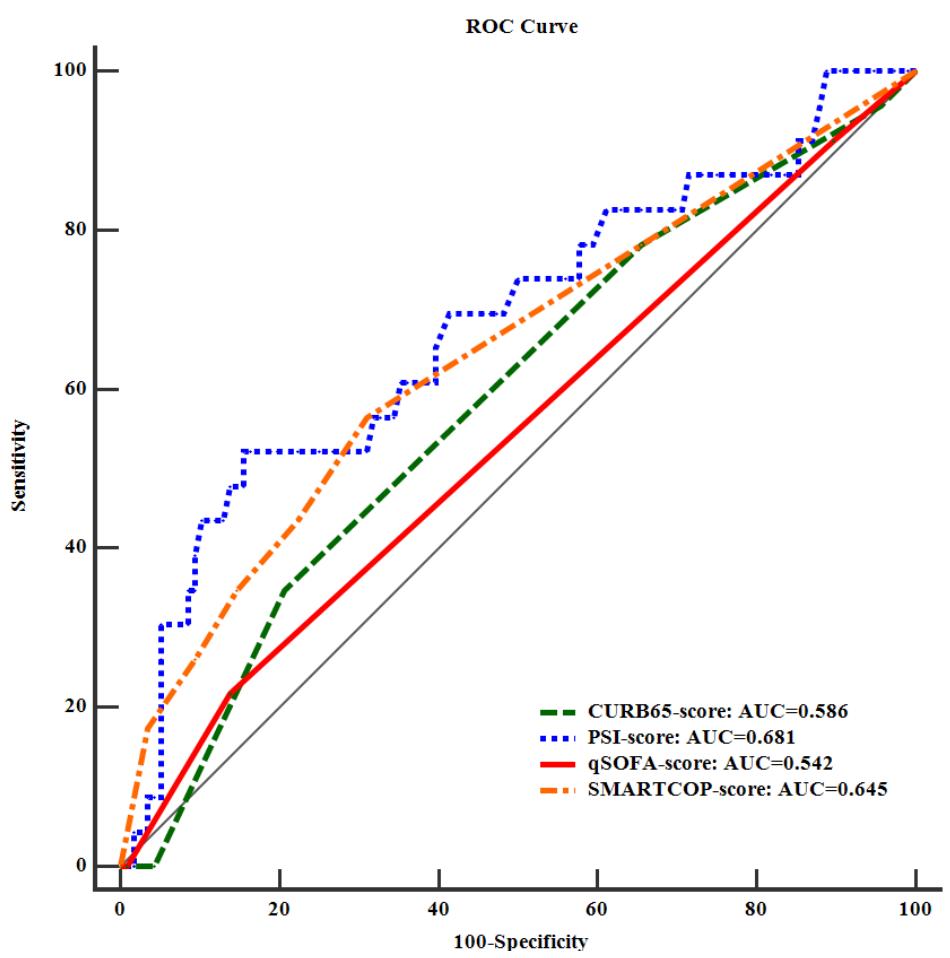

Fig. 1. Receiver operating characteristic curves for PSI, CURB-65, SMART-COP and qSOFA scores for adverse outcome in CAP patients 


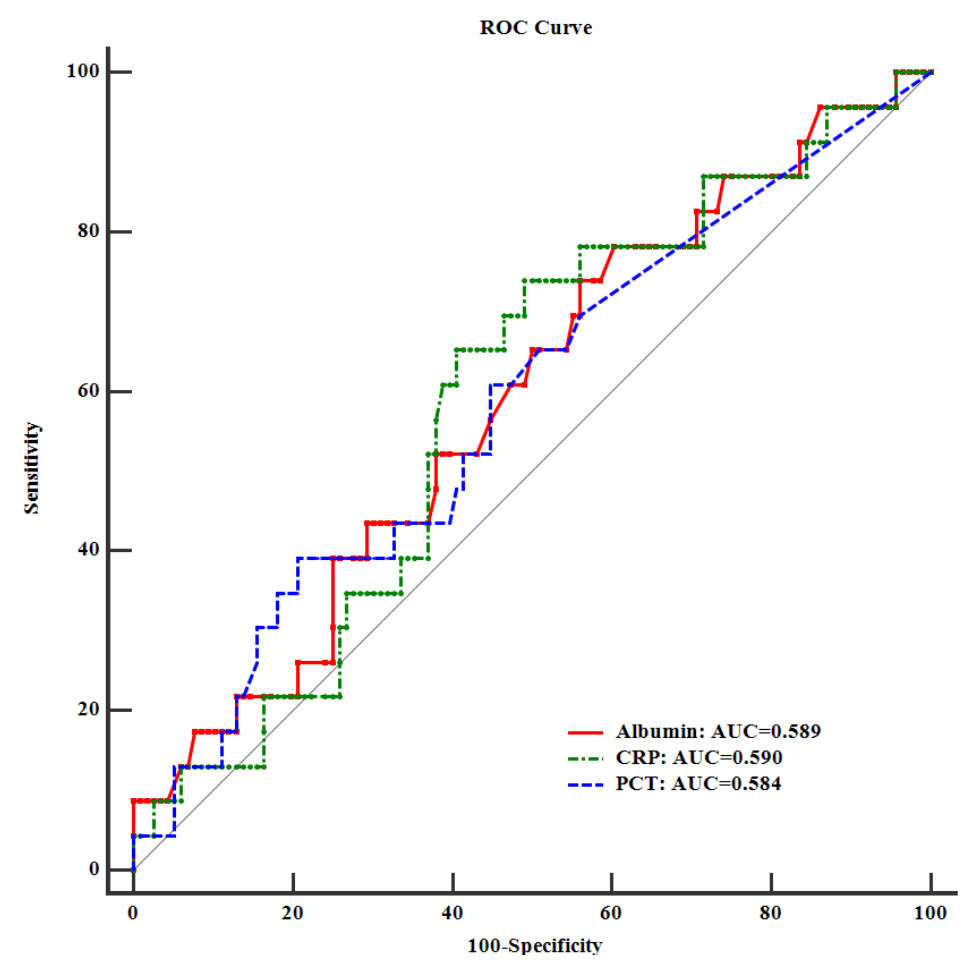

Fig. 2. Receiver operating characteristic curves for PCT, CRP and low albumin levels for adverse outcome in CAP patients

With regard to the biomarkers used, the AUC for CRP, PCT and serum albumin were $0.590(\mathrm{p}=$ $0.152), 0.584(\mathrm{p}=0.199)$ and $0.589(\mathrm{p}=0.168)$, respectively (Figure 2).

\section{Discussion}

In our research, we found a higher incidence among males and the elderly, similarly to others (15). Our study population had a mean LOS of 7.35 days, which was a little shorter than in most similar cohorts in Europe. The mean LOS for CAP patients in Europe and in the United States of America differs greatly $(13,16,17)$. Rozenbaum M. et al registered a mean LOS of 6.7 days, compared to Garau J. et al who found a mean LOS of 11.5 days. Unlike other reports, we found the etiology in only $24 \%$ of cases and that is mainly due to a relatively high percentage of contaminated sputum collected from patients and because of the use of antibiotics before admission in $23.7 \%$ of cases.

CAP is known to be the leading cause of sepsis, so early management is based on severity assessment tools. To predict severity at admission, we used CURB-65, PSI, SMART-COP and qSOFA scoring systems. The majority of our patients were included in PSI class risk 4 and 5 and CURB-65 group 2 and 3, but only 2 patients died $(1.4 \%)$ during the hospital stay. Although mortality rates are higher in the PSI class risk $5(27-31 \%)$ (3), and in the CURB-65 group 3 (30\%) (4), our patients had a favourable outcome. This is partly explained by the fact that there are many elderly patients, with multiple comorbidities, which results in higher points on severity scores.

Regarding the need for IRVS calculated with SMART-COP, most of our cases posed low risk. 
Only 21 cases were considered to be at high risk of in-hospital mortality using the qSOFA score. We found that the qSOFA score correlates with other severity scores in CAP, meaning CURB-65 and SMART-COP, but not with PSI. The similarities between the CURB-65 and the qSOFA scores can be explained by the fact that they have comparable parameters, although the hypotension and respiratory rate thresholds for CURB -65 were stricter than those for qSOFA.

The PSI score is recognized in patients with CAP as the best predictor of mortality, but it is considerably more complex than the other severity scores, because it uses more variables. Nonetheless, it is not used adequately in clinical practice, as physicians find it hard to use (18). Chen et al (19) stated that qSOFA was better than CRB-65 in identifying high mortality risk individuals and pneumonia patients requiring ICU admission. In our research, qSOFA was also used to predict adverse outcomes in CAP patients and was compared with CURB-65, PSI and SMART-COP. AUROC for qSOFA was lower than for PSI and SMART-COP, close to CURB-65. However, only PSI and SMART-COP reached statistical significance, reinforcing the idea that PSI is still the best severity predictor in patients with CAP. In a retrospective study including 1545 patients, Dosanjh D. et al concluded that CURB-65 and qSOFA can both stratify according to risk on 30-day mortality, but none are particularly precise (19). Tokioka F. et al found that qSOFA's prognostic performance for in-hospital mortality and admission to ICU was not substantially different from CURB-65 and PSI (21). Similarly, our results demonstrated that the qSOFA score did not outperform PSI and SMART-COP scores and it was similar to CURB-65 regarding adverse outcome. One possible explanation is that our mortality level was very low. The same results were reported by Ahnert P. et al in a recent study (22).
All severity scores in the category of patients with qSOFA $\geq 2$ were found to be higher than those with scores $<2$. Similarly, Asai N. et al (11) proved that the predictive value of the SOFA score was superior to other predictive scores for CAP patients with a qSOFA score $\geq 2$. A qSOFA score $\geq 2$ may therefore be associated with pneumonia severity.

CRP and PCT have become the most extensively tested in CAP as useful methods for diagnosis, prognosis and follow-up treatment (23). We used CRP and PCT levels in order to see if value at admittance can assess disease severity and we found correlations between PCT and all of the severity scores used and between CRP and most of the scores, with the exception of PSI. Naderi HR et al. found that PCT levels were substantially higher in patients with severe pneumonia PSI class $\geq 4$, CURB- $65 \geq 3$ and SMART-COP $\geq 3$ (24). Chalmers JD et al. demonstrated a strong correlation between CRP during hospital stays and 30-day mortality, the need for mechanical ventilation and/or vasopressor support, CRP being an independent CAP severity marker (25). We found no correlation between CRP and the PSI score, although we did find associations between CRP and CURB 65, SMART-COP and qSOFA scores. Somehow similarly to us, Thiem $\mathrm{U}$ et al. also found no relationship between CRP and PSI (26). CRP and PCT levels were higher in the qSOFA score $\geq 2$, than in the qSOFA score $<2$, meaning that their concentrations increase with severity.

Serum albumin levels play an important role in the maintenance of normal colloid osmotic pressure, the transport of endogenous components and the scavenging of oxidizing agents (27). Hypoalbuminemia's role in causing a poor prognosis in an illness is largely associated with malnutrition and mostly seen in elderly hospitalized patients. However, systemic inflammatory response can decrease serum albumin levels (28). Studies in CAP have shown that non-survivors 
had lower albumin levels than survivors (24). In our study, low albumin levels were correlated with all severity scores. When we compared albumin levels in the group with qSOFA score of $<2$ and the group with qSOFA score of $\geq 2$, we observed, similarly to others, a significant difference in albumin levels with increasing severity of CAP. In 3463 patients hospitalized with CAP, Viasus D et al. found that the lower the serum albumin levels were, the higher the risk of complications was, and that low albumin levels were independently associated with a poor outcome, within 24 hours of admission (29). In a large cohort of CAP patients, Magnussen B et al. found that the level of plasma albumin was a strong mortality predictor for CAP patients, and that hypoalbuminemia was caused by factors related to the acute response (30). When we searched for the ability of the studied biomarkers to predict adverse outcome, none of them reached statistical significance, probably because the number of patients included was small and the adverse outcomes were seen in only $16.5 \%$ of patients. Our study has some limitations. First of all, the study included a limited number of patients from one hospital. A broad multicentre study is required to assess the accuracy of the qSOFA score and other severity scores as prognostic tools for adverse outcomes among CAP patients. Secondly, our study included patients with a lot of comorbidities that could be a bias for assessment scores. Another limitation is that we determined CRP, PCT and albumin levels only at admittance and not in dynamic.

\section{Conclusions}

In conclusion, our study showed that the qSOFA score- a much simpler to use score- was able to evaluate the severity of CAP, similarly to other severity scores for CAP. All severity scores and biomarkers in the category of patients with a qSOFA of $\geq 2$ were higher than those with scores of $<2$. Serum albumin may be a cheap, easily available marker that can be correlated with the severity of CAP. Several studies are needed to assess the prediction of these biomarkers and severity scores in pneumonia, regarding adverse outcome.

\section{Abbreviations}

CAP-Community-acquired pneumonia HCAP- Healthcare associated pneumonia IDSA- Infectious Diseases Society of America ACCP/SCCM- American College of Chest Physicians/ Society of Critical Care Medicine LOS-length of stay PSI- Pneumonia Severity Index

CURB- C-confusion; U- blood urea; $\mathrm{R}=$ respiratory frequency; B- Blood pressure

SMART-COP- S-systolic blood pressure, Mmultilobar involvement, A- serum albumin, R-respiratory rate, T- tachycardia; C- confusion, O- oxygen saturation, $\mathrm{P}-\mathrm{pH}$

qSOFA - quick sequential organ failure assessment

ICU- intensive care unit

IRVS- Intensive respiratory and vasopressor support

CRP-C-reactive protein

PCT- procalcitonin

ROC- receiver operating characteristic

\section{Authors' contribution}

TRE- Conceptualization, Investigation, Formal Analysis, Writing, Project Administration

CV- Supervision, Validation, Project Administration

LM- Writing-review, Supervision, Validation, Project Administration

\section{Conflict of interest}

There was no conflict of interest in this work. 


\section{References}

1. Antunes G, Evans SA, Lordan JL, Frew AJ. Systemic cytokines levels in community-acquired pneumonia and their association with disease severity. Eur Respir J. 2002 Oct;20 (4):990-5. DOI: 10.1183/09031936.02.00295102

2. Community-Acquired Pneumonia (CAP): Practice Essentials, Overview, Etiology of Community-Acquired Pneumonia [Online]. [2017] [cited February 2020]; Available from: URL: https://emedicine.medscape. com/article/234240-overview.

3. Fine MJ, Auble TE, Yealy DM, Hanusa BH, Weissfeld LA, Singer DE, et al. A prediction rule to identify lowrisk patients with community-acquired pneumonia. $\mathrm{N}$ Engl J Med. 1997 Jan;336(4):243-50. DOI: 10.1056/ NEJM199701233360402

4. Lim WS, van der Eerden MM, Laing R, Boersma WG, Karalus N, Town GI, et al. Defining community acquired pneumonia severity on presentation to hospital: an international derivation and validation study. Thorax. 2003 May;58(5):377-82. DOI: 10.1136/tho$\operatorname{rax} .58 .5 .377$

5. [Online]. 2019 [cited February 2020]. Available from: URL: https://lifeinthefastlane.com/wp-content/uploads/2010/07/etg-smartcop.

6. Almirall J, Bolibar I, Toran P, Pera G, Boquet X, Balanzo X, et al. Contribution of C-reactive protein to the diagnostic and assessment of severity of community-acquired pneumonia. Chest. 2004 Apr;125(4):1335-42. DOI: 10.1378/chest.125.4.1335

7. Christ-Crain M, Stolz D, Bingisser R, Muller C, Miedlinger D, Huber PR, et al. Procalcitonin guidance of antibiotic therapy in community-acquired pneumonia: a randomized trial. Am J Respir Crit Care Med. 2006 Jul;174(1):84-93. DOI: 10.1164/rccm.200512-1922OC

8. Magnussen B, Oren Gradel K, Gorm Jensen T, Kolmos HJ, Pedersen C, Just Vinholt P, et al. Association between Hypoalbuminaemia and Mortality in Patients with Community-Acquired Bacteraemia Is Primarily Related to Acute Disorders. PLoS ONE. 2016 Sep;11(9): e0160466. DOI: 10.1371/journal. pone. 0160466

9. Singer M, Deutschman CS, Seymour CW, Shankar-Hari M, Annane D, Bauer M, et al. The third International Consensus definitions for Sepsis and Septic Shock (Sepsis-3). JAMA. 2016 Feb;315(8):801-10. DOI: 10.1001/jama.2016.0287

10. Raith EP, Udy AA, Bailey M, McGloughlin S, MacIsaac C, Bellomo R, et al. Prognostic accuracy of SOFA score, SIRS criteria and qSOFA score for in-hospital mortality among adults with suspected infection admitted to the Intensive Care Unit. JAMA. 2017 Jan;317(3):290-300. DOI: 10.1001/jama.2016.20328

11. Asai N, Watanabe H, Shiota A, Kato H, Sakanashi D, Hagihara M, et al. Efficacy and accuracy of q SOFA and SOFA scores as prognostic tools for community-acquired and healthcare- associated pneumonia. Int J Infect Dis. 2019 Jul;84:89-96. DOI: 10.1016/j. ijid.2019.04.020

12. Tripon RE, Neagoe IB, Budisan L, Pop TL, Cristea V, Stanca LM, et al. Systemic interleukins levels in community-acquired pneumonia and their association with adverse outcomes. Rev Romana Med Lab. 2019;27(2):189-98. DOI: 10.2478/rrlm-2019-0020

13. Metlay JP, Waterer GW, Long AC, Anzueto A, Brozek J, Crothers K, et al. Diagnosis and treatment of adults with community-acquired pneumonia. An official clinical practice guideline of the American Thoracic Society and Infectious Diseases Society of America. Am J Respire Crit Care Med. 2019 Oct;200(7):e45-e67. DOI: 10.1164/rccm.201908-1581ST

14. Bone R, Balk R, Cerra F, Dellinger R, Fein A, Knaus W, et al. Definitions for Sepsis and Organ Failure and Guidelines for the Use of Innovative Therapies in Sepsis. The ACCP/SCCM Consensus Conference Committee. Amrican College of Chest Physicians/Society of Critical Care Medicine. Chest. 1992 Jun;101(6):164455. DOI: 10.1378/chest.101.6.1644

15. Gutiérrez F, Masiá M, Mirete C, Soldán B, Carlos Rodríguez J, Padilla $\mathrm{S}$, et al. The influence of age and gender on the population-based incidence of community-acquired pneumonia caused by different microbial pathogens. J Infect. 2006 Sep;53(3):166-74. DOI: 10.1016/j.jinf.2005.11.006

16. Rozenbaum M, Mangen M, Huijts S, van der Werf T, Postma M. Incidence, direct costs and duration of hospitalization of patients hospitalized with community acquired pneumonia: A nationwide retrospective claims database analysis. Vaccine. 2015 Jun;33(28):3193-9. DOI: $10.1016 /$ j.vaccine.2015.05.001

17. Garau J, Baquero F, Pérez-Trallero E, Pérez J, Martín-Sánchez A, García-Rey C, et al. Factors impacting on length of stay and mortality of community-acquired pneumonia. Clin Microbiol Infect. 2008 Apr;14(4):322-9. DOI: 10.1111/j.14690691.2007.01915.x

18. Song H, Moon GH, Kim HS. Efficacy of quick SOFA with lactate concentration for predicting mortality in patients with community-acquired pneumonia in the emergency department. Clin Exp Emerg Med. 2019 Mar;6(1):1-8. DOI: 10.15441/ceem.17.262

19. Chen XY, Wang JY, Guo SB. Use of CRB-65 and quick SOFA to predict site of care and mortality in pneumonia patients in the emergency department: a retrospective study. Crit Care. 2016 Jun;20(1):167. DOI: 10.1186/ s13054-016-1351-0

20. Dosanjh DPS, Grudzinska F, Aldridge K, Hughes S, Thickett D. Risk stratification in community acquired pneumonia-CURB65, SIRS or qSOFA? A retrospec- 
tive analysis. Thorax. 2017 Dec;72(Suppl 3): A8.2-A9. DOI: 10.1136/thoraxjnl-2017-210983.14

21. Tokioka F, Okamoto H, Yamazaki A, Itou A, Ishida T. The prognostic performance of qSOFA for community acquired pneumonia. J Intensive Care. 2018 Aug;6:46. DOI: 10.1186/s40560-018-0307-7

22. Anhert P, Creutz P, Horn K, Schwarzenberger F, Kiehntopf $M$, Hossain $H$, et al. Sequential organ failure assessment score in an excellent operationalization of disease severity of adult patients with hospitalized community acquired pneumonia- results from the prospective observational PROGRESS study. Crit Care. 2019 Apr;23:110. DOI: 10.1186/s13054-019-2316-X

23. Shaddock EJ. How and when to use common biomarkers in community-acquired pneumonia. Pneumonia (Nathan). 2016 Oct;8:17. DOI: 10.1186/s41479-0160017-7

24. Naderi HR, Sheybani F, Sarvghad M, Nooghabi MJ. Can procalcitonin add to the prognostic power of the severity scoring system in adults with pneumonia? Tanaffos. 2015;14(2):95-106.

25. Chalmers JD, Singanayagam A, Hill AT. C-reactive protein is an independent predictor of severity in community-acquired pneumonia. Am J Med. 2008 Mar;121(3):219-25. DOI: 10.1016/j.am- jmed.2007.10.033

26. Thiem U, Niklaus D, Sehloff B, Stuckle C, Heppner HJ, Endres HG, et al. C-reactive protein, severity of pneumonia and mortality in elderly, hospitalised patients with community-acquired pneumonia. Age Ageing. 2009 Nov;38(6):693-7. DOI: 10.1093/ageing/afp164

27. Nicholson JP, Wolmarans MR, Park GR. The role of albumin in critical illness. Br J Anaesth. 2000 Oct;85(4):599-610. DOI: 10.1093/bja/85.4.599

28. Lee JH, Kim J, Kim K, Jo YH, Rhee J, Kim TY, et al. Albumin and $\mathrm{C}$-reactive protein have prognostic significance in patients with community-acquired pneumonia. J Crit Care. 2011 Jun;26(3):287-94. DOI: 10.1016/j.jcrc.2010.10.007

29. Viasus D, Garcia-Vidal C, Simionetti A, Manresa F, Dorca J, Gudoil F, et al. Prognostic value of serum albumin levels in hospitalized adults with community-acquired pneumonia. J Infect. 2013 May;66(5):415-23. DOI: 10.1016/j.jinf.2012.12.007

30. Magnussen B, Oren Gradel K, Gorm JT, Kolmos HJ, Pedersen C, Just Vinholt P, et al. Association between hypoalbuminemia and mortality in patients with community-acquired bacteraemia is primarily related to acute disorders. PLos One. 2016 Sep;11(9):e0160466. DOI: 10.1371/journal.pone.0160466 